\title{
SoundDuctFlow: A Modelica Library for Modeling Acoustics and Flow in Duct Networks
}

\author{
Helmut Kühnelt Thomas Bäuml Anton Haumer \\ Austrian Institute of Technology, Mobility Department \\ Giefinggasse 2, A-1210 Vienna, Austria \\ \{helmut.kuehnelt,thomas.baeuml,anton.haumer\}@ait.ac.at
}

\section{Abstract}

SoundDuctFlow, a Modelica library for the joint calculation of acoustic and flow quantities in HVAC (Heating, Ventilation and Air Conditioning) ducts is presented. Modeling the sound propagation in ducts by one dimensional acoustic twoport methods is a well-established technique for the acoustic characterization of large HVAC duct networks. Two different approaches of acoustic modeling will be considered in the framework: When resonant phenomena are insignificant, it is often sufficient to apply band-averaged models to predict the sound power level and the transmission loss within the individual components of the duct network. For the low frequency range where linear plane wave propagation is valid, acoustic two and multi-port models based on a transmission matrix formulation of the sound pressure can be applied for high frequency resolution and phase accurate calculations. For the prediction of the mean air flow in the duct network pressure loss models are applied. The coupling of acoustic and flow elements permits the simulation of flow acoustic phenomena.

For setting up large networks a smooth work flow is vital for the user: The simulation is easily set up using the GUI provided by Dymola. External parameterisation ensures persistent data management. The resulting system of equations is automatically pre-processed and solved by Dymola.

In this paper an overview of this new library is given together with exemplary applications.

Keywords: acoustics; flow; ducts; flow noise; HVAC; modeling; simulation; Modelica library

\section{Introduction}

The joint calculation of sound level, air flow rate and pressure loss in large HVAC duct networks as well as the prediction of the noise level in the passenger compartment gains more and more in importance in the early phase of design. The unified Modelica framework for one-dimensional acoustic and flow simulation introduced here serves as a tool for concept modeling. Used by the design engineer in an early design phase, it has to meet several requirements: The prediction method has to be fast and computational efficient. All kinds of available data should be used, from analytic and (semi)empiric models to data obtained from measurements and three-dimensional acoustic and fluid dynamics computer simulations to characteristic diagrams and sparse point data provided by component manufacturers. It also should be extendable to add physical phenomena like heat transfer or transport of humid air and complex flow-acoustic interactions. Finally the framework should be open for integration into a complete system simulation.

\section{Modeling acoustics in duct networks}

In modeling acoustics of duct networks usually following assumptions are made: Only the steady state of the system has to be regarded. The dimensionality of the systems often can be reduced from 3-D to 1-D. The acoustic properties of each component can be characterized by an acoustical two-port (or a multi-port in the case of a branching), a grey box whose transfer behavior can be calculated by means of 1-D to 3-D methods, like finite (FEM) or boundary element methods (BEM), 
independently from each other. Two standard one-dimensional approaches are available:

First, the sound power-based description has been widely used and forms the basis of the most standards and guidelines for the analysis of sound in ducts, e.g. see ASHRAE [1, 2] or VDI [3]. It can be applied for frequencies well above the plane wave cut-off. The sound power level within a duct network can be determined by summation of the losses and gains in sound power level at the connecting interfaces of the components from the fan towards the terminal sections of the network. All contributions resulting from wave reflections are neglected in this approach. This makes the prediction procedure very simple, but reduces the reliability considerably.

Second, the plane wave based description of ducts, mufflers and networks $[4,5,6]$ covers the low frequency range. A variety of analytical plane wave two-port models is available in the literature. The acoustical transmission matrix of non-standard components can be determined by 3-D FEM/BEM or unsteady CFD simulations in case of non-zero mean flow. In HVAC duct networks with their large lateral dimensions, however, the frequency range of plane wave propagation is rather limited. Nevertheless, accurate sound prediction at lower frequencies below a few hundred $\mathrm{Hz}$ is quite important, since absorbing liners are ineffective at those frequencies. In Table 1 the plane wave cutoff frequency, $f_{c}$, is given for some exemplary circular ducts.

Table 1: Plane wave cutoff frequencies for circular ducts.

\begin{tabular}{|c|c|c|}
\hline diameter & $f_{c}$ & typical usage \\
\hline $10 \mathrm{~mm}$ & $20 \mathrm{kHz}$ & brass wind instrument \\
$50 \mathrm{~mm}$ & $4 \mathrm{kHz}$ & car exhaust pipe \\
$300 \mathrm{~mm}$ & $670 \mathrm{~Hz}$ & HVAC ducts \\
\hline
\end{tabular}

\subsection{Sound power-based description}

The sound power-based description of sound transmission in ducts is valid for frequencies well above the plane wave cut-off. Wave reflections are neglected. The transmission loss of a duct element is considered. The variable of state then is the sound power level $L_{W}$ :

$$
L_{W}=10 \log _{10}\left(\frac{P_{a c}}{P_{0}}\right) \mathrm{dB}
$$

with a reference sound power level $P_{0}=10^{-12}$ Watt. Typically $L_{W}$ is specified for octave or $1 / 3-$ octave frequency bands. The summation of levels $L_{i}$ obeys the following rule:

$$
L_{\text {sum }}=10 \log _{10} \sum_{i=1}^{n} 10^{L_{i} / 10}
$$

The acoustic elements based on the sound power description are extended from one, two or multiports. Each port is represented by a signalbased connector holding two arrays of incoming and outgoing $L_{W}$ in $N$ frequency bands. Since there are no reflections considered, in passive elements like silencers, each incoming $L_{W}$ signal is mapped onto the outgoing $L_{W}$ independently of its counterpart:

$$
\begin{aligned}
& L_{W i A}^{\text {out }}=L_{W i B}^{i n}-\Delta L_{W i B \rightarrow A} \\
& L_{W i B}^{\text {out }}=L_{W i A}^{i n}-\Delta L_{W i A \rightarrow B}
\end{aligned}
$$

with $\Delta L_{W i}$ the reduction of the sound power level at the $i$ th band. This allows bidirectional usage of the components.

In-duct sources, like fans or bends contributing regenerated flow-noise, add their forward and backward contribution to the total sound pressure level according to eq. (2)

Figure 1 gives some examples of acoustic components already included in SoundDuctFlow, like different types of pipes, bends, silencers, fans.

\subsection{Plane wave description}

In the wave based description the steady state, frequency dependent plane wave solution of the wave equation is regarded. This approach is valid only for the low frequency region below the plane wave cut off. Although this region is rather limited because of the large lateral dimensions usually found in HVAC duct networks, an accurate calculation is nevertheless important, since absorbing materials are rather ineffective at those frequencies. Two physically equivalent methods can be applied to characterize the individual duct element.

In the scattering matrix method the sound pressure $p$ is decomposed into forwards and backwards traveling plane waves:

$p(x, t)=\hat{p}(x) \exp ^{\mathrm{i} \omega t}=p^{+} \exp ^{\mathrm{i}(\omega t-k x)}+p^{-} \exp ^{\mathrm{i}(\omega t+k x)}$ 


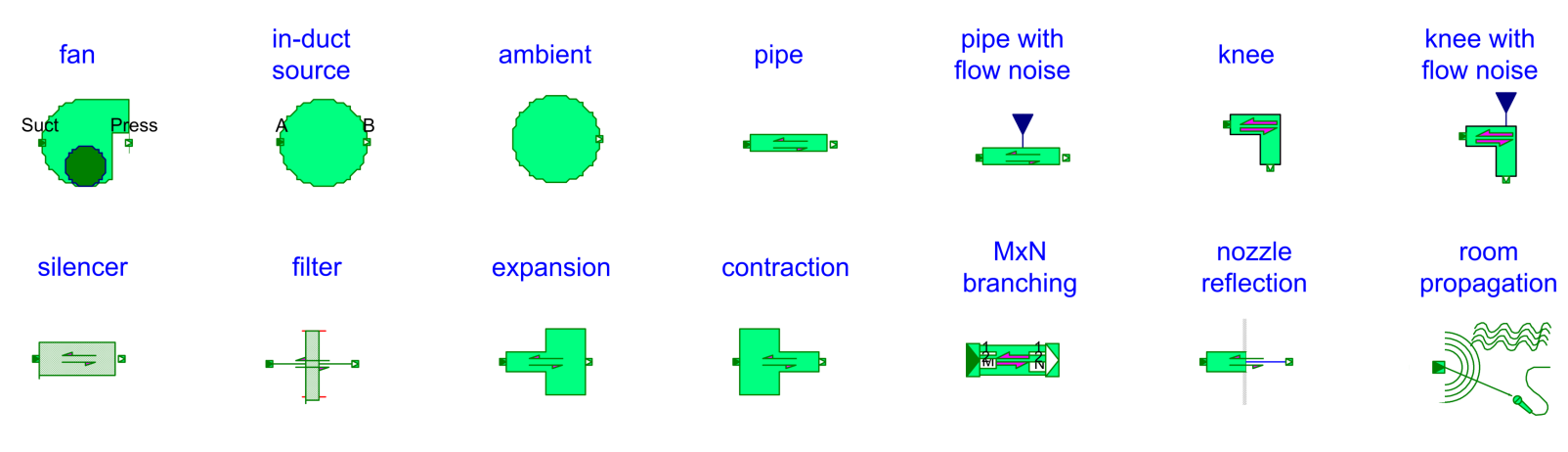

Figure 1: Examples of acoustic elements.

where $k=\omega / c_{0}, p^{+}$and $p^{-}$are the amplitudes of forwards and backwards traveling waves. The acoustic particle velocity then is:

$$
\hat{v}=\frac{1}{\rho_{0} c_{0}}\left(p^{+} \exp ^{-\mathrm{i} k x}-p^{-} \exp ^{\mathrm{i} k x}\right) .
$$

A four pole scattering matrix $S$ maps the forward and backward propagating sound pressures of one port onto the second port:

$$
\left(\begin{array}{l}
p_{1}^{+} \\
p_{1}^{-}
\end{array}\right)=\left(\begin{array}{ll}
S_{11} & S_{12} \\
S_{21} & S_{22}
\end{array}\right)\left(\begin{array}{l}
p_{2}^{+} \\
p_{2}^{-}
\end{array}\right)
$$

This formulation is preferred in connection with the experimental determination of the acoustic reflection coefficient $R=p^{-} / p^{+}$at the interfaces of duct elements.

The transmission matrix method describes the relation between acoustic pressure, $p$, and acoustic volume flow, $q$, in forward and backward sections of the single element by the matrix $T$ :

$$
\left(\begin{array}{l}
p_{1} \\
q_{1}
\end{array}\right)=\left(\begin{array}{ll}
T_{11} & T_{12} \\
T_{21} & T_{22}
\end{array}\right)\left(\begin{array}{l}
p_{2} \\
q_{2}
\end{array}\right)
$$

This formulation is preferred for modeling in Modelica since the potential variable $p$ and the flow variable $q$ directly can be used to constitute the physical connector.

A collection of plane wave models will be included into the SoundDuctFlow library.

\subsection{Modeling of junctions}

In the sound power-based description, at junctions the incident sound power is distributed over the total of all outgoing duct sections. By definition there are no reflections. The proportion of the sound power transmitted from section $i$ to section $j, W_{i \rightarrow j}$, then is

$$
W_{i \rightarrow j}=W_{i} \frac{S_{j}}{S_{t o t}-S_{i}}
$$

where $W_{i}$ is the sound power incident at the duct section $i, S_{i}, S_{j}$ and $S_{t o t}$ are the incident, outgoing and total cross-sectional areas.

In the plane wave model, the reflection of waves at a multiple junction is considered. As an example a T-shaped junction between three pipes of cross-sectional surfaces $A_{1}, A_{2}$ and $A_{3}$ is examined here. From the equation of Bernoulli we find that the acoustic pressures $p_{1}$ and $p_{2}$ in the duct just before and after the side-branch have to be the same as the pressure $p_{3}$ at the mouth of the side-branch:

$$
p_{1}=p_{2}=p_{3}
$$

The conservation of mass yields that the sum of the acoustic volume flows at the junction is zero:

$$
q_{1}+q_{2}+q_{3}=0
$$

\section{Modeling flow and pressure loss in duct networks}

The 1-d modeling of air flow in HVAC ducts and other components is based on several approximations: The air flow in HVAC ducts with its low Mach number is regarded as incompressible, steady state and fully stabilized. In most cases it is also turbulent. The state variables for fluid flow are the static pressure as the potential variable and the mass flow rate as the flow variable. The pressure loss coefficient $K=\frac{\Delta P}{\rho U^{2} / 2}$ relates the pressure difference $\Delta P$ between the two ports of an flow duct element to the kinetic energy density of the flow. It depends on the Reynolds 


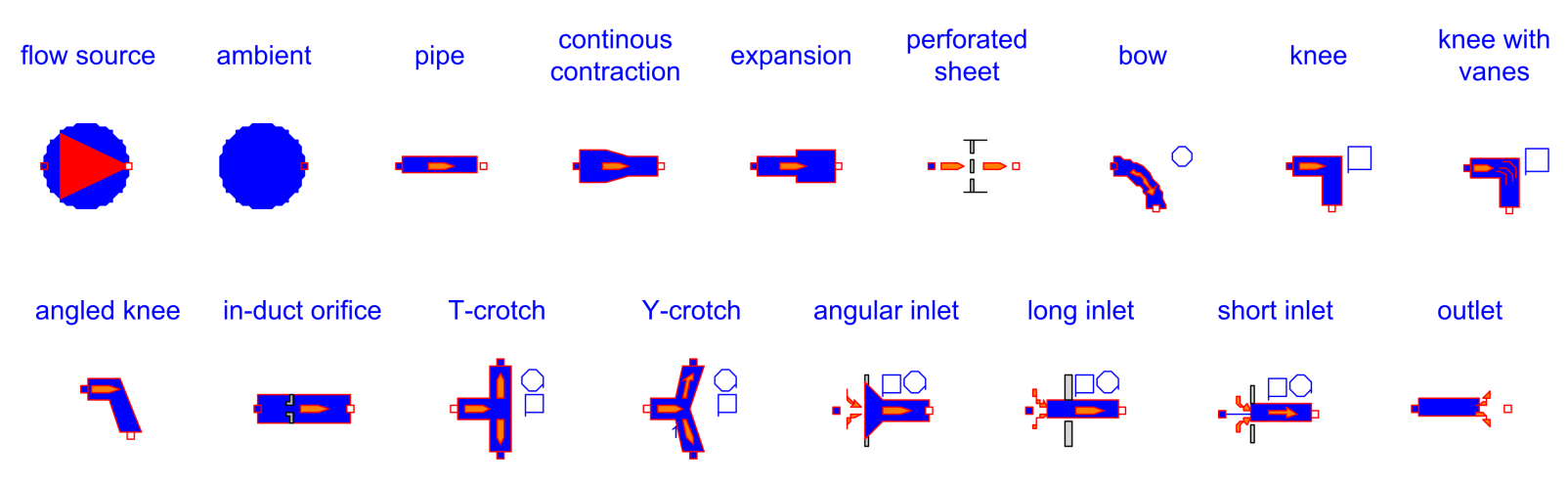

Figure 2: Examples of flow elements.

number, on the relative roughness and on the cross-sectional shape of the duct.

Analytic models, like the Colebrook-White equation, as well as measurement data, given as characteristic diagram or interpolated formula $[7,8,9]$ or tabulated manufacturer data serves as input for the various flow models. Effects by turbulence, adverse velocity gradients, crosssectional shape, surface roughness, curvature, interaction between elements in not fully developed flow regions result in corrective terms. Within the Modelica library flow elements for standard duct components are available (Fig. 2).

\section{Complex duct components}

Complex compound flow-acoustic duct components (see Fig. 3) can be assembled from simple elements representing either the acoustic or the flow part of a duct element using a special connector that combines the acoustic and the flow connector. This allows the modeling of nonstandard components.

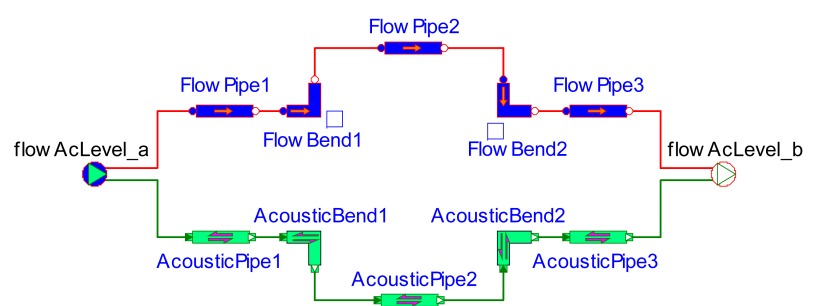

Figure 3: Complex compound flow-acoustic duct component utilizing a combined flow-acoustic connector.

\section{Flow-acoustic interaction}

Flow-acoustic coupling can also be modeled on this basis. Inside duct elements, like bends or orifices, the high Reynolds number flow triggers flow noise. In most cases the back-reaction of the sound field on the flow is negligibly weak. This effect can be modeled by extracting the state of the fluid flow and feeding it into the acoustic element. There the additional flow noise is calculated by (semi)empirical models, as given in $[3,10]$ for instance. An example of a complex compound pipe component with flow noise is given in Fig. 4.

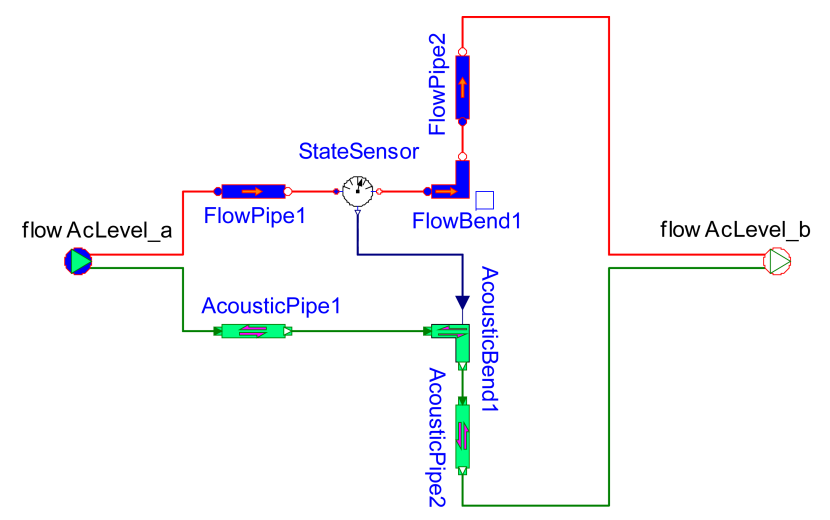

Figure 4: Compound flow-acoustic duct component in which the mean flow velocity generates flow noise at the bend.

\section{Work flow and practical issues}

When dealing with large models, the parameterisation of each component in the Dymola GUI is error-prone and not very convenient for the user, 


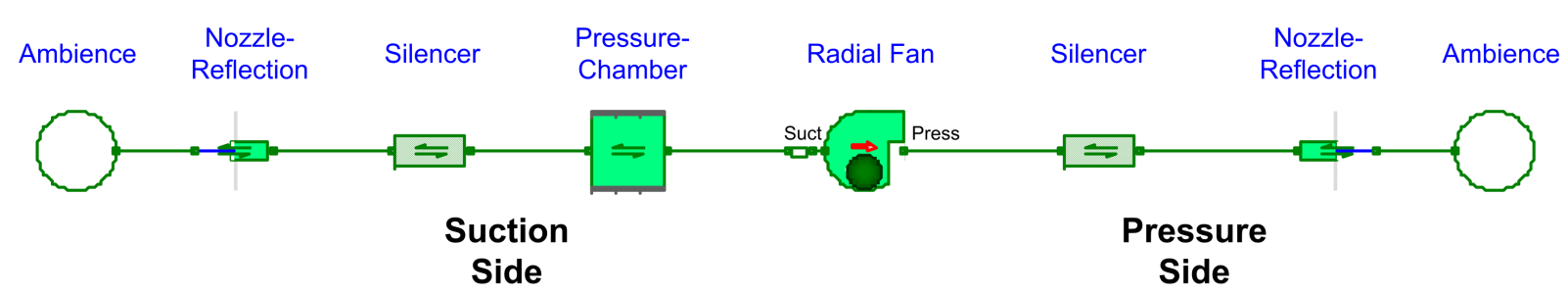

Figure 5: Acoustic representation of of a ventilation unit.

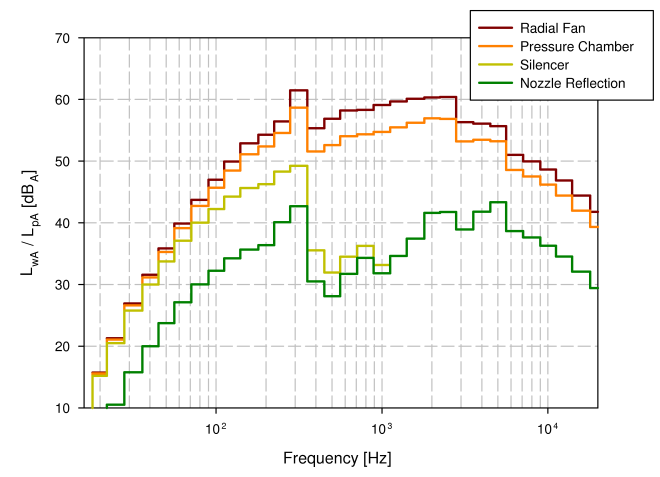

(a)

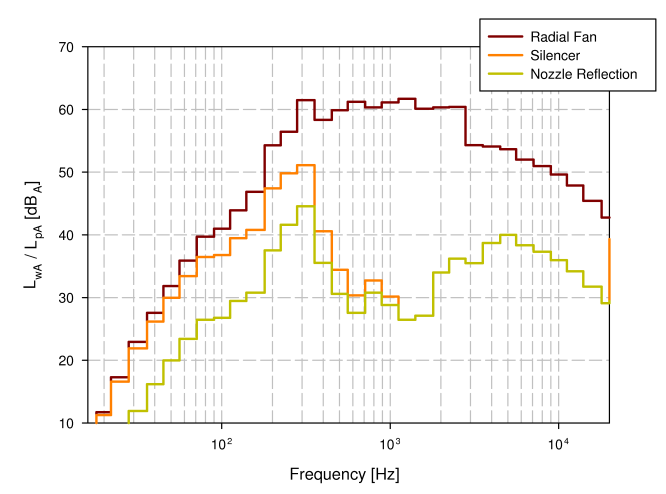

(b)

Figure 6: A-weighted $L_{W}$ in third octave bands at the suction side (a) and pressure side (b) components of a ventilation unit.

especially when the parameters are varied repeatedly within the design process. Therefore we use an external parameterisation procedure to ensure consistent data handling and a smooth work flow: After setting up the model in the Dymola GUI, all model parameters and connections are extracted into an XML structure. This XML structure then is filled in with the parameters' numerical values and read in at the run time of the simulation. All results are also written to an XML file for external postprocessing procedures like report generation.

\section{Examples}

As a first example, the acoustic representation of a ventilation unit is presented in Fig. 5. Here it it is not connected to the duct network but in a configuration for measuring the radiated sound power from its exits. Therefore the nozzle reflections at the intake and exhaust pipe terminations have to be included. The predicted A-weighted $L_{W}$ at each element is shown in Fig. 6. Prediction and measurement of the A-weighted $L_{W}$ at the exits (Table 2) agree quite well.

Table 2: Measured and predicted A-weighted sound power level of a ventilation unit.

\begin{tabular}{|c|c|c|}
\hline \multirow{2}{*}{ Position } & \multicolumn{2}{|c|}{ Sound power level $L_{w A}$} \\
\cline { 2 - 3 } & Measurement & Prediction \\
\hline Pressure Side & $52 \mathrm{~dB}(\mathrm{~A})$ & $50.4 \mathrm{~dB}(\mathrm{~A})$ \\
Suction Side & $54 \mathrm{~dB}(\mathrm{~A})$ & $51.9 \mathrm{~dB}(\mathrm{~A})$ \\
\hline
\end{tabular}

The ventilation unit is then installed in a small duct network (Fig. 7). The predicted third band SPL at each element is shown in Fig. 8.

\section{Conclusions and perspectives}

The concepts of sound propagation in HVAC systems together with modeling of the air flow are realized in the new Modelica library SoundDuctFlow, providing a joint prediction of the noise level and the pressure loss in the duct network. The concept of acoustical connectors based either 


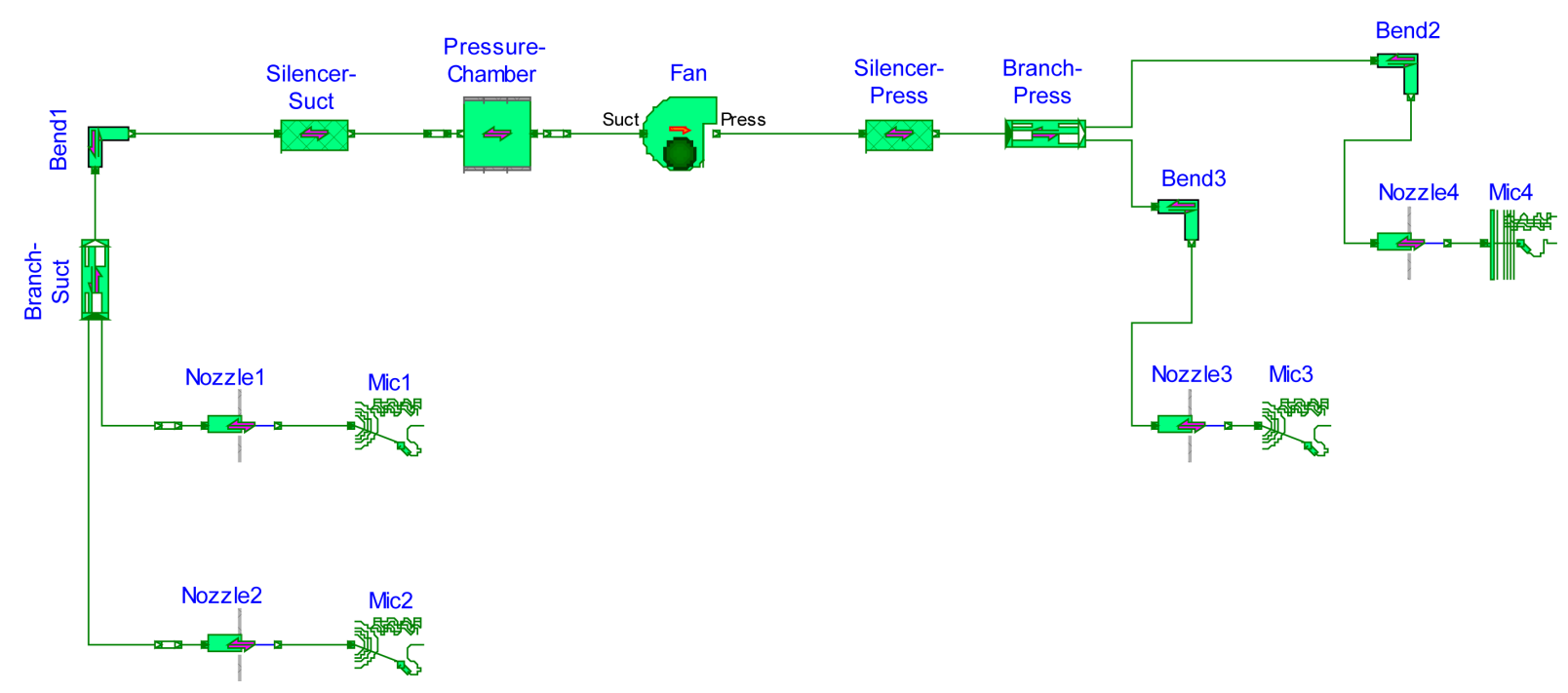

Figure 7: Acoustic representation of a ventilation duct network.

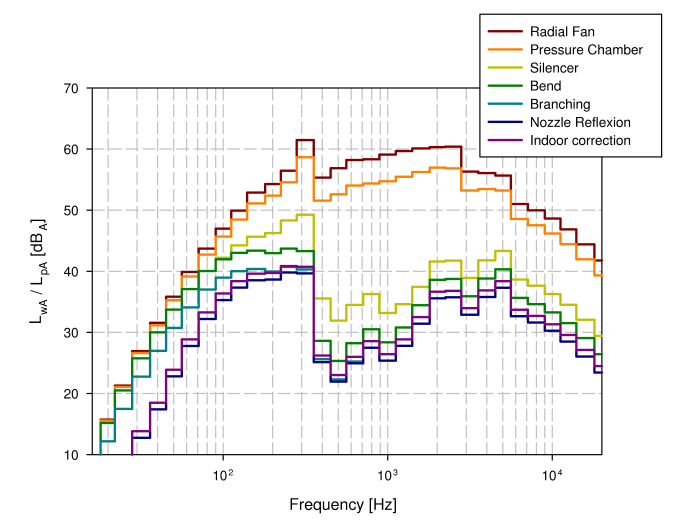

(a)

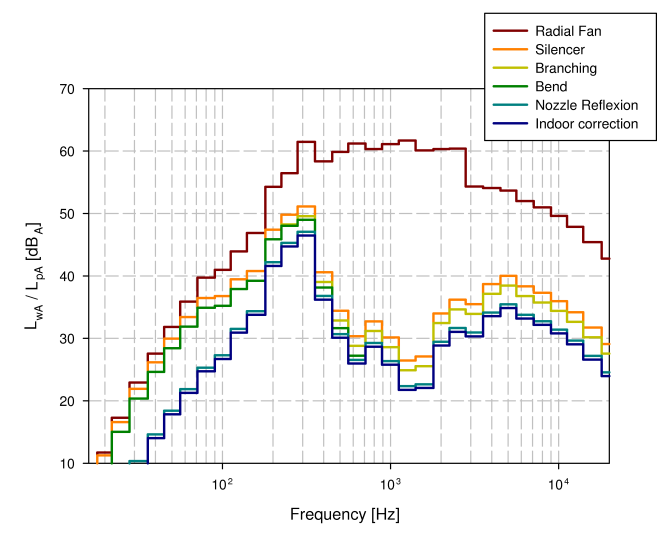

(b)

Figure 8: A-weighted $L_{W}$ at each element of a ventilation duct network along two paths from the fan to a listener, (a) at the suction side and (b) the pressure side.

on a sound power level description or a plane wave description was discussed. The effects of the geometric conditions on the mean air flow is modeled by $1 \mathrm{D}$ pressure loss models. The triggering of noise by flow effects is also modeled. The modeling of the emitted noise level of a ventilation unit agrees quite well with acoustical measurements.

SoundDuctFlow will be extended to incorporate also heat transfer, transport of humid air and complex flow-acoustic interactions. For the last point, however, there will be the need for new models deduced from complex 3D computational aeroacoustic simulations.

\section{Acknowledgments}

We are very grateful to Gerhard Karlowatz, Liebherr-Transportation Systems, Austria, for providing real life network examples and measurement results. 


\section{References}

[1] Reynolds, D. D. and Bledsoe, J. M., Algorithms for HVAC Acoustics, ASHRAE Inc., Atlanta, 1991.

[2] ASHRAE, Sound and Vibration Control, 2007 ASHRAE Handbook - HVAC Applications, ASHRAE Inc., Atlanta, 2007 (Chapter 47).

[3] VDI, Association of German Engineers, VDI Guideline 2081, VDI, Düsseldorf, 2001.

[4] Munjal, M. L., Acoustics of Ducts and Mufflers, Wiley, New York, 1987.

[5] Boden, H. and Abom, M., Modelling of Fluid Machines as Sources of Sound in Duct and Pipe Systems, Acta Acustica, 3 (1995), 545560.

[6] Boden, H. and Glav, R., Exhaust and Intake Noise and Acoustical Design of Mufflers and Silenecers, in Handbook of Noise and Vibration Control, ed. by Crocker, M. J., John Wiley \& Sons, 2007.

[7] Miller, D. S., Internal Flow Systems, BHR Group Limited, Cranfield, UK, 1996 (2nd ed).

[8] Idelchik, I.E., Handbook of Hydraulic Resistance, Begell House, 1996 (3rd ed).

[9] Recknagel, Sprenger, Schramek, Taschenbuch für Heizung und Klimatechnik. R. Oldenbourg Verlag, München Wien, 1999 (69th ed).

[10] Nelson, P. A. and Morfey, C. L., Aerodynamic sound production in low speed flow ducts, Journal of Sound and Vibration, 79(1981), 263-289. 